\title{
Comparison of platelet reactivity between prasugrel and ticagrelor in patients with acute coronary syndrome: a meta-analysis
}

Mingxiang Wen ${ }^{1 *+} \mathbb{D}$, Yaqi $\mathrm{Li}^{2+}$, Xiang $\mathrm{Qu}^{2+}$, Yanyan $\mathrm{Zhu}^{3}$, Lingfang $\mathrm{Tian}^{4}$, Zhongqin Shen ${ }^{4}$, Xiulin Yang ${ }^{2}$ and Xianqing $\mathrm{Shi}^{1 *}$

\begin{abstract}
Background: This meta-analysis aimed to compare the effects of prasugrel and ticagrelor on high (HTPR) and low on-treatment platelet reactivity (LTPR) in patients with acute coronary syndrome (ACS).

Methods: Eligible studies were retrieved from PubMed, Embase, and the Cochrane Library. HTPR and LTPR were evaluated on the basis of the vasodilator-stimulated phosphoprotein platelet reactivity index (VASP-PRI) and P2Y12 reaction units (PRUs). HTPR and LTPR were analyzed using risk ratios (RRs) and their $95 \%$ confidence intervals (Cls). Weighted mean difference (WMD) and 95\% Cl were used to calculate the pooled effect size of platelet reactivity (PR).

Results: Fourteen eligible studies were obtained, which included 2629 patients treated with ticagrelor $(n=1340)$ and prasugrel $(n=1289)$. The pooled results showed that the prasugrel-treated patients had higher platelet reactivity than the ticagrelor-treated patients (PRU: WMD $=-32.26 ; 95 \% \mathrm{Cl}$ : -56.48 to $-8.76 ; P<0.01$; VASP-PRI: WMD $=-9.61 ; 95 \% \mathrm{Cl}$ : -14.63 to $-4.60 ; P=0.002$ ). No significant difference in HTPR based on PRU was identified between the ticagrelor and prasugrel groups $(P=0.71)$, whereas a lower HTPR based on VASP-PRI was found in the ticagrelor-treated patients than in the prasugrel-treated patients ( $R R=0.30 ; 95 \% \mathrm{Cl}: 0.12-0.75 ; P=0.010$ ). In addition, the results showed a lower LTPR was observed in the prasugrel group than in the ticagrelor group ( $\mathrm{RR}=1.40 ; 95 \% \mathrm{Cl}: 1.08-1.81 ; P=0.01)$.

Conclusions: Prasugrel might enable higher platelet reactivity than ticagrelor. Ticagrelor could lead to a decrease in HTPR and increase in LTPR. However, this result was only obtained in pooled observational studies. Several uncertainties such as the nondeterminancy of the effectiveness of ticagrelor estimated using VASP-PRI or the definition of HTPR (a high or modifiable risk factor) might have affected our results.
\end{abstract}

Keywords: Ticagrelor, Prasugrel, Acute coronary syndrome, Meta-analysis

\section{Background}

Acute coronary syndrome (ACS), a common but serious type of coronary artery disease [1], is characterized by primary atherosclerotic plaque rupture and secondary completely or partially occlusive thrombus that leads to

\footnotetext{
*Correspondence: Wenmingxiang168@163.com; 364888849@qq.com ${ }^{\dagger}$ Mingxiang Wen, Yaqi Li and Xiang Qu contributed equally to this work. ${ }^{\dagger}$ Mingxiang Wen and Yaqi Li are co-first authors.

'Intensive Care Unit, Guizhou Provincial People's Hospital, No. 58 Zhongshan East Road, Nanming District, Guiyang 550002, Guizhou, China

Full list of author information is available at the end of the article
}

ST-segment elevation myocardial infarction (STEMI), non-STEMI (NSTEMI), and unstable angina [2]. Percutaneous coronary intervention $(\mathrm{PCI})$ is considered the preferred treatment for ACS to prevent thrombotic cardiovascular events [3].

Dual antiplatelet therapy with aspirin and a P2Y12 blocker is currently considered the primary treatment for ACS patients undergoing PCI [4]. Clopidogrel is the most common P2Y12 blocker used in dual antiplatelet therapies; however, its delayed action, susceptibility to

(C) The Author(s). 2020 Open Access This article is licensed under a Creative Commons Attribution 4.0 International License, which permits use, sharing, adaptation, distribution and reproduction in any medium or format, as long as you give appropriate credit to the original author(s) and the source, provide a link to the Creative Commons licence, and indicate if changes were made. The images or other third party material in this article are included in the article's Creative Commons licence, unless indicated otherwise in a credit line to the material. If material is not included in the article's Creative Commons licence and your intended use is not permitted by statutory regulation or exceeds the permitted use, you will need to obtain permission directly from the copyright holder. To view a copy of this licence, visit http://creativecommons.org/licenses/by/4.0/. The Creative Commons Public Domain Dedication waiver (http://creativecommons.org/publicdomain/zero/1.0/) applies to the data made available in this article, unless otherwise stated in a credit line to the data. 
genetic polymorphisms, and significant inter-individual response variability limit its clinical efficacy in patients with ACS [5]. The novel P2Y12 receptor antagonists, prasugrel and ticagrelor, are clinically superior to clopidogrel in patients with ACS who have undergone PCI $[4,6]$. Several prospective clinical studies have compared the therapeutic effects of prasugrel and ticagrelor on platelet reactivity (PR) in patients with ACS [7-21]. Schüpke et al. [22] reported that the incidence of death, myocardial infarction, or stroke was significantly lower in prasugrel-treated patients with ACS than in ticagrelor-treated. In addition, Alexopoulos et al. [8] demonstrated that ticagrelor induced a significantly higher platelet inhibition than prasugrel in patients with ACS treated with PCI. These studies mainly focused on the efficacy of the two antiplatelet agents. A subsequent meta-analysis compared the two treatments on the basis of high on-treatment platelet reactivity (HTPR) and revealed that ticagrelor had a lower HTPR than prasugrel [23]. Although previous meta-analysis has reported the effect of ticagrelor and prasugrel on PR [24], the main purpose of their article is to investigate the effects of different detection methods on the inhibition of platelet response of the two drugs. However, the effects of prasugrel and ticagrelor on HTPR and low on-treatment platelet reactivity (LTPR) in patients with ACS have not been systematically reported. Therefore, an integrative meta-analysis of the published results is necessary.

In the present study, we compared the effect of both treatments on HTPR and LTPR in patients with ACS. The evaluation criteria for $\mathrm{PR}$ were based on the vasodilator-stimulated phosphoprotein PR index (VASP$\mathrm{PRI}$ ) and P2Y12 reaction units (PRUs).

\section{Methods}

\section{Search strategy}

This study was performed in accordance with the Preferred Reporting Items for Systematics reviews and Meta-Analysis (PRISMA) guidelines [25]. No review protocol for this meta-analysis was registered before the study was undertaken. PubMed, Embase, and the Cochrane Library were searched for all the studies comparing ticagrelor and prasugrel treatments in patients with ACS that were published through February 11, 2020. The following search string was used: "prasugrel" AND "ticagrelor" AND "acute coronary syndrome" OR "ACS." The references of the acquired articles were manually searched to identify more potential studies. Only English language articles were included.

\section{Selection criteria}

All the included articles met the following criteria: (1) included patients with ACS; (2) compared the therapeutic effects of prasugrel and ticagrelor; and (3) outcomes contained HTPR, LTPR, or PR. Notably, the main end point of this meta-analysis was PR, which was detected by VerifyNow-P2Y12 function assay or VASP test. According to the standards of the literature, PR was divided into PRU and PRI.

The exclusion criteria were as follows: (1) retrospective analysis, review article, conference abstract, or protocol; (2) duplicate search result; (3) therapeutic effects of prasugrel or ticagrelor compared with those of other drugs; (4) included healthy individuals; (5) required outcomes not reported; (6) drug utilization study only identified medical expenses; or (7) preclinical study.

\section{Data extraction and quality assessment}

The data were extracted independently by two authors (Xiang Qu and Yanyan Zhu) and included the following information: first author name, publication date, study type, study period, patient age and sex, sample size, therapeutic strategy, clinical presentation, study duration, testing standard, definitions of HTPR and LTPR, and outcome indicators (HTPR, LTPR, and PR). Disagreements were resolved by discussion with the corresponding author (Mingxiang Wen). Furthermore, the article quality of randomized controlled trials (RCTs) was verified using the Cochrane risk of bias assessment tool, while non-randomized cohort studies were evaluated using the Newcastle-Ottawa Scale (NOS) [26].

\section{Statistical analysis}

The statistical analysis was performed using the RevMan 5.3 software. For categorical data (HTPR and LTPR), risk ratios (RRs) and their 95\% confidence intervals (CIs) were set as evaluation indexes. For continuous data (PR), the weighted mean difference (WMD) and its 95\% CIs were used to calculate the pooled effect size. In addition, for multiple assessments of residual PR, we selected the data of the last measurement as the principal analysis. Owing to the large differences in clinical and methodological data in the included studies, a random effects model was applied to estimate the combined effect size. Cochran's Q test and the $I^{2}$ index were used to quantify the degree of heterogeneity [27]. Briefly, $P$ values of < 0.05 and/or $I^{2}$ values of $>50 \%$ were considered indicative of significant heterogeneity; otherwise, differences were considered non-significant. In addition, to investigate potential sources of heterogeneity, a subgroup analysis was performed in terms of study type (RCT or cohort), testing time $(<24 \mathrm{~h}$ after loading dose or 5-30 days after treatment initiation), and special population (patients with STEMI or diabetes). Finally, the publication bias of the included studies was detected using Egger's test. 


\section{Results}

\section{Data retrieval}

A flowchart of the literature search and selection process is shown in Fig. 1. A total of 2030 studies were identified from PubMed (773), Embase (949), and the Cochrane Library (308) in accordance with the initial search strategy. Of the studies, 1920 irrelevant studies and 42 repetitive studies were excluded. Among the remaining 68 studies, 39 were removed (details are shown in Fig. 1). Thereafter, 15 articles (three reviews, two protocols, three uncorrected groupings, four without interested outcomes, and one with different evaluation criteria) were excluded. Finally, 14 eligible studies were included in this meta-analysis [7-9, 11-19, 21, 28].

\section{Characteristics of the included studies}

The detailed characteristics of the 14 included studies are summarized in Table 1 . The publication dates of these studies ranged from 2012 to 2017. Among these studies, 10 were RCTs and four were prospective cohort studies, which included a total of 2629 patients with ACS treated with ticagrelor $(n=1340)$ or prasugrel $(n=1289)$.

\section{Quality assessment}

The results of the Cochrane risk of bias assessment showed that all 10 RCTs were of high quality (Fig. 2a) but did not describe the group allocation concealment process. The risk of bias was low for random sequence generation and blinding of outcome assessments in all 10 RCTs. Only in the study by Franchi et al. [14], the participants and researcher were not blinded (Fig. 2b). The NOS scores of the included cohort studies ranged from 5 to 8 , implying that all were of high quality (Table 2).

\section{PR assessment}

PR was evaluated on the basis of the PRU and VASPPRI values. The PRU values were obtained using the VerifyNow P2Y12 assay (VN-P2Y12; Accumetrics Inc., San Diego, CA, USA), while the VASP values were evaluated using a commercially available kit (VASP; Biocytex, Marseille, France) and performed using a flow cytometer. Notably, the evaluation criteria in our analysis were as follows: PRUs of $\geq 208$ or $\geq 230$ and VASPPRI values of $>50 \%$ were defined as HTPR, whereas

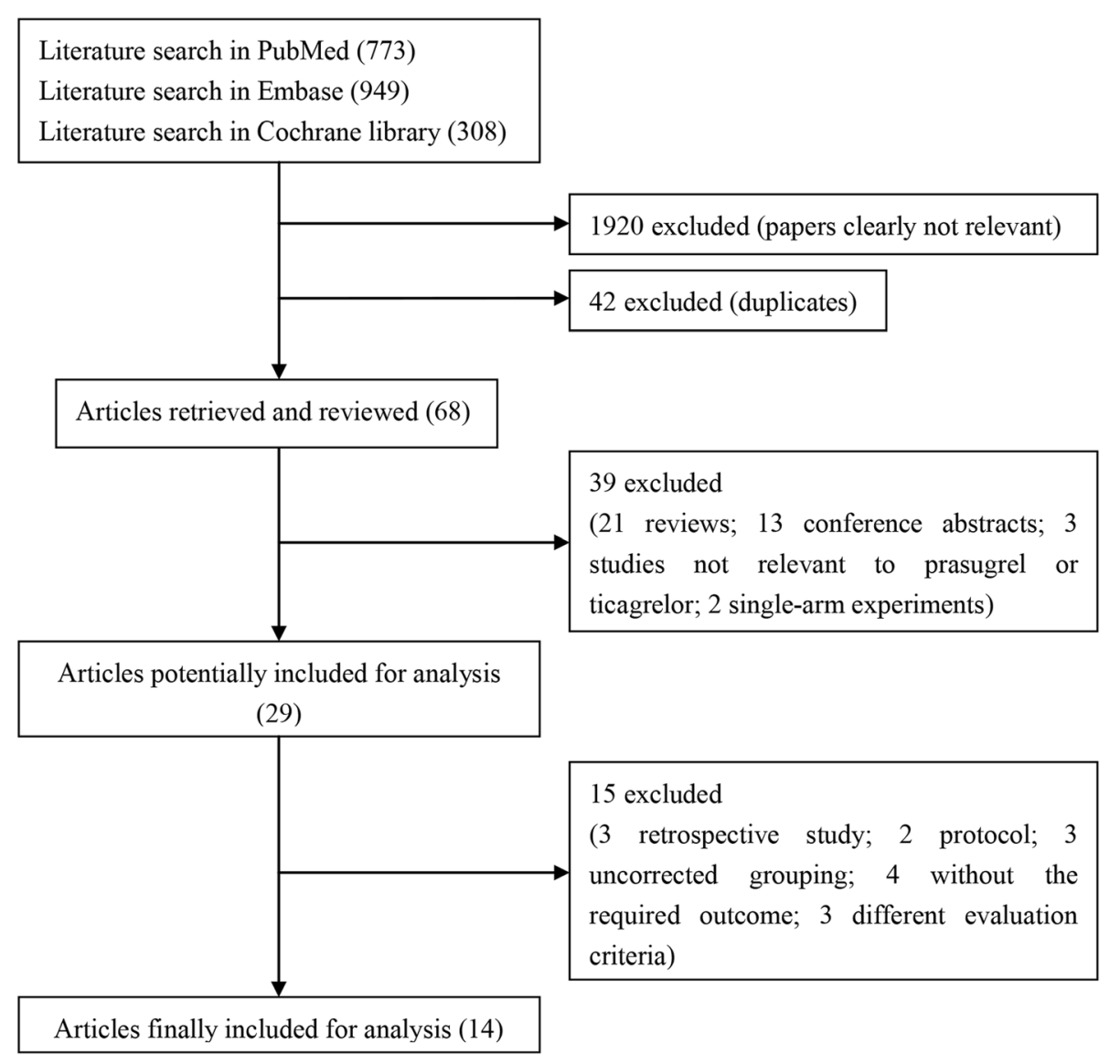

Fig. 1 Flowchart of the data retrieval process 


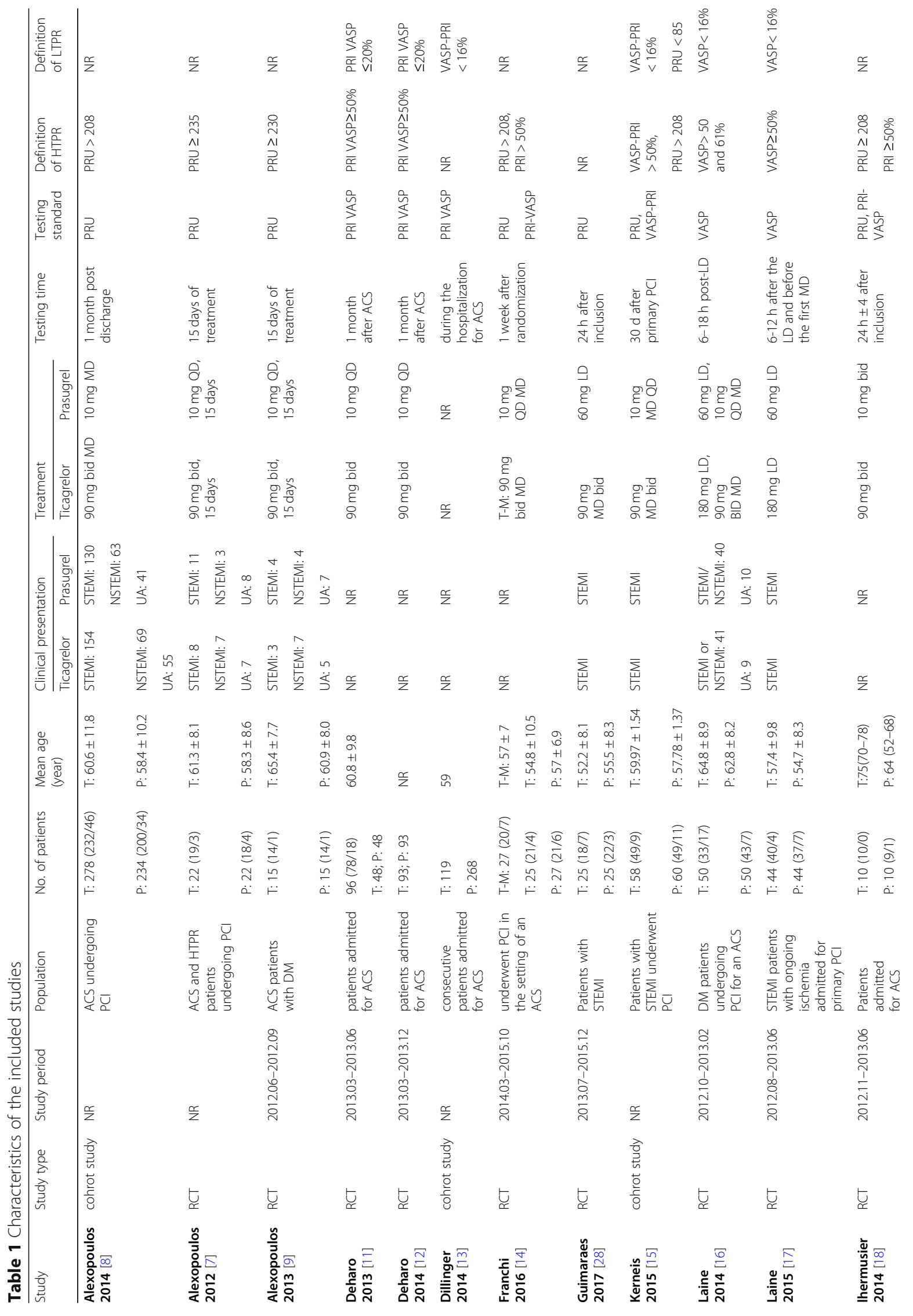




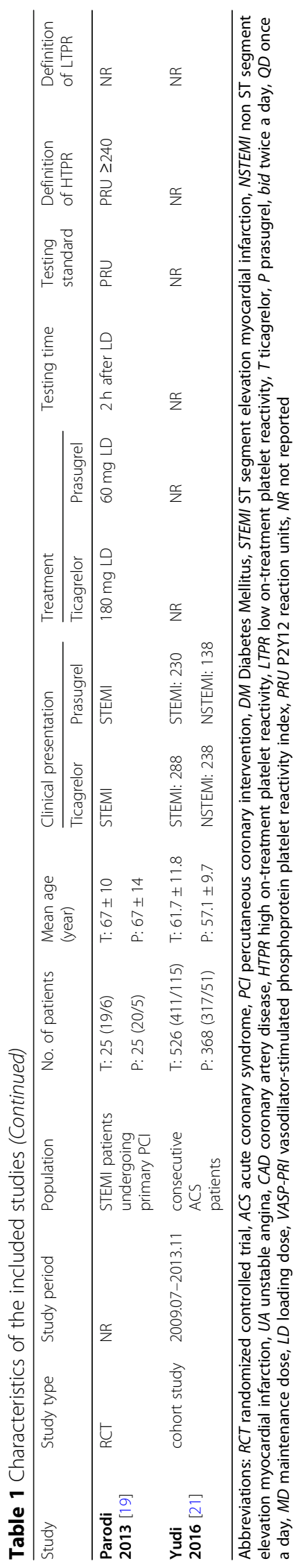




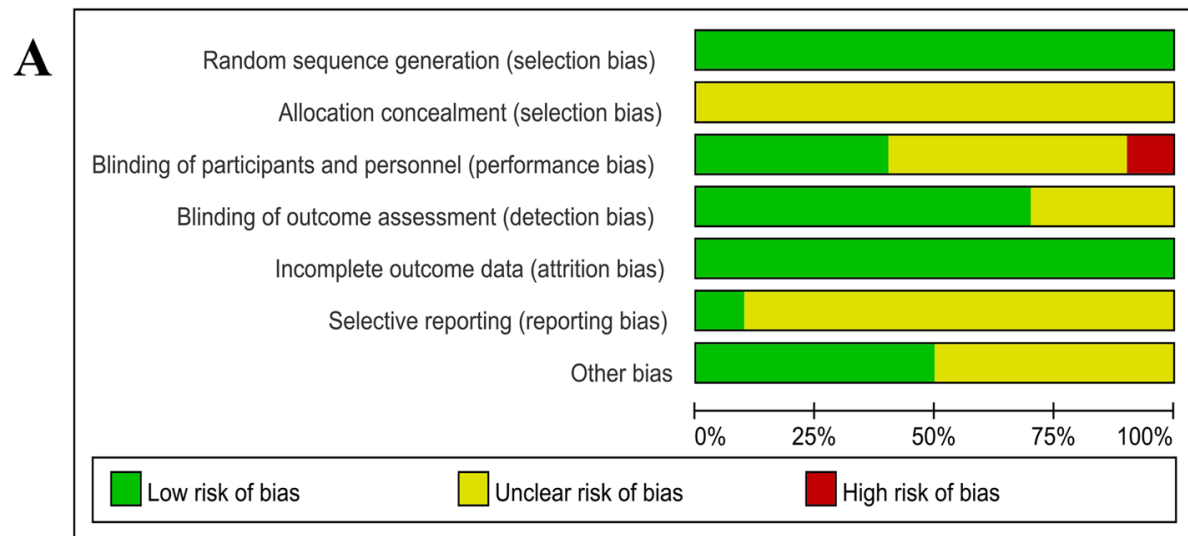

\section{B}

\begin{tabular}{|c|c|c|c|c|c|c|c|}
\hline Alexopoulos 2012 & + & $?$ & + & + & + & $?$ & + \\
\hline Alexopoulos 2013 & + & $?$ & + & + & + & + & + \\
\hline Deharo 2013 & + & $?$ & $?$ & + & + & $?$ & $?$ \\
\hline Deharo 2014 & + & $?$ & $?$ & $?$ & + & $?$ & $?$ \\
\hline Franchi 2016 & + & $?$ & 0 & + & + & $?$ & $?$ \\
\hline Guimaraes 2017 & + & $?$ & $?$ & $?$ & + & $?$ & + \\
\hline Ihermusier 2014 & + & $?$ & $?$ & + & + & $?$ & $?$ \\
\hline Laine 2014 & + & $?$ & + & + & + & $?$ & + \\
\hline Laine $2014-2$ & + & $?$ & $?$ & $?$ & + & $?$ & $?$ \\
\hline Parodi 2013 & + & $?$ & + & + & + & $?$ & + \\
\hline
\end{tabular}

Fig. 2 Quality assessment of the included studies. a Risk (\%) of bias among the included studies. Green represents low risk of bias; yellow, unclear risk of bias; and red, high risk of bias. $\mathbf{b}$ Risk of bias items among the 10 included studies. +,?, and -indicate low, unclear, and high risk of bias, respectively 
Table 2 Quality assessment of the included cohort studies ${ }^{\mathrm{a}}$

\begin{tabular}{|c|c|c|c|c|c|c|c|c|c|}
\hline Cohort & $\begin{array}{l}\text { Representativeness } \\
\text { of the exposed } \\
\text { cohort }\end{array}$ & $\begin{array}{l}\text { Selection of } \\
\text { the unexposed } \\
\text { cohort }\end{array}$ & $\begin{array}{l}\text { Ascertainment } \\
\text { of exposure }\end{array}$ & $\begin{array}{l}\text { Outcome of } \\
\text { interest not } \\
\text { present at } \\
\text { start of study }\end{array}$ & $\begin{array}{l}\text { Control for } \\
\text { important } \\
\text { factor or } \\
\text { additional } \\
\text { factor }^{b}\end{array}$ & $\begin{array}{l}\text { Outcome } \\
\text { assessment }\end{array}$ & $\begin{array}{l}\text { Follow-up long } \\
\text { enough for } \\
\text { outcomes to } \\
\text { occur }\end{array}$ & $\begin{array}{l}\text { Adequacy of } \\
\text { follow-up } \\
\text { of cohorts }\end{array}$ & $\begin{array}{l}\text { Total } \\
\text { quality } \\
\text { scores }\end{array}$ \\
\hline $\begin{array}{l}\text { Alexopoulos } \\
2014 \text { [8] }\end{array}$ & $\hat{s}$ & $\sum$ & 㶦 & $\dot{s}$ & 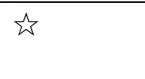 & 约 & 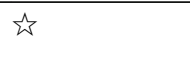 & 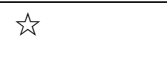 & 8 \\
\hline $\begin{array}{l}\text { Dillinger } \\
2014 \text { [13] }\end{array}$ & $i s$ & $\sum$ & 㶦 & $\hat{z}$ & 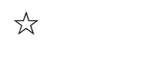 & $i$ & $i$ & $\sum$ & 8 \\
\hline $\begin{array}{l}\text { Kerneis } \\
2015 \text { [15] }\end{array}$ & $i s$ & $\dot{z}$ & 弥 & - & $i s$ & $i$ & $i s$ & 访 & 7 \\
\hline Yudi 2016 [21] & is & $\hat{s}$ & 岤 & 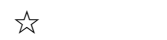 & - & 项 & - & - & 5 \\
\hline
\end{tabular}

${ }^{\text {a }}$ A study could be awarded a maximum of one star for each item except for the item Control for important factor or additional factor

${ }^{\mathrm{b}}$ A maximum of 2 stars could be awarded for this item. Studies that controlled for cardiovascular disease received one star, whereas studies that controlled for other important confounders such cancer received an additional star

c A cohort study with a follow-up rate $>75 \%$ was assigned one star

VASP-PRI values of $<16 \%$ or PRUs of $<85$ were defined as LTPR.

Among the included cohort studies, the pooled risk ratio from the combined data of the seven PRU studies showed that prasugrel treatment had a higher PR $(\mathrm{WMD}=-32.62 ; 95 \% \mathrm{CI}:-56.48$ to $-8.76 ; P<0.01$; Fig. 3a) than ticagrelor treatment, and heterogeneity was observed between studies $(P<0.01)$. Moreover, the pooled analysis of VASP-PRI indicated that the PR in the prasugrel group was significantly higher than that in the ticagrelor group (WMD $=-8.06$; $95 \% \mathrm{CI}:-12.98$ to $-3.14 ; P=0.001$; Fig. 3b). A significant heterogeneity was observed, with a $P$ value of $<0.01$ and $I^{2}$ of $87 \%$.
Furthermore, to examine the sources of heterogeneity, a subgroup analysis was performed. In the PRU group, a subgroup analysis according to study type revealed that the PR in the prasugrel group was significantly higher than that in the ticagrelor group in the cohort studies $(\mathrm{WMD}=-37.55 ; 95 \% \mathrm{CI}:-65.66$ to $-9.44 ; P=0.009)$. Meanwhile, significant differences were observed at 530 days after treatment initiation (WMD $=-36.72$; 95\% CI: -57.04 to $-16.40 ; P<0.01)$. In the PRI group, the $\mathrm{PR}$ in the prasugrel group was markedly higher than that in the ticagrelor group in the RCT and cohort. The included studies were classified according to testing time $(<24 \mathrm{~h}$ after the loading dose and 5-30 days after

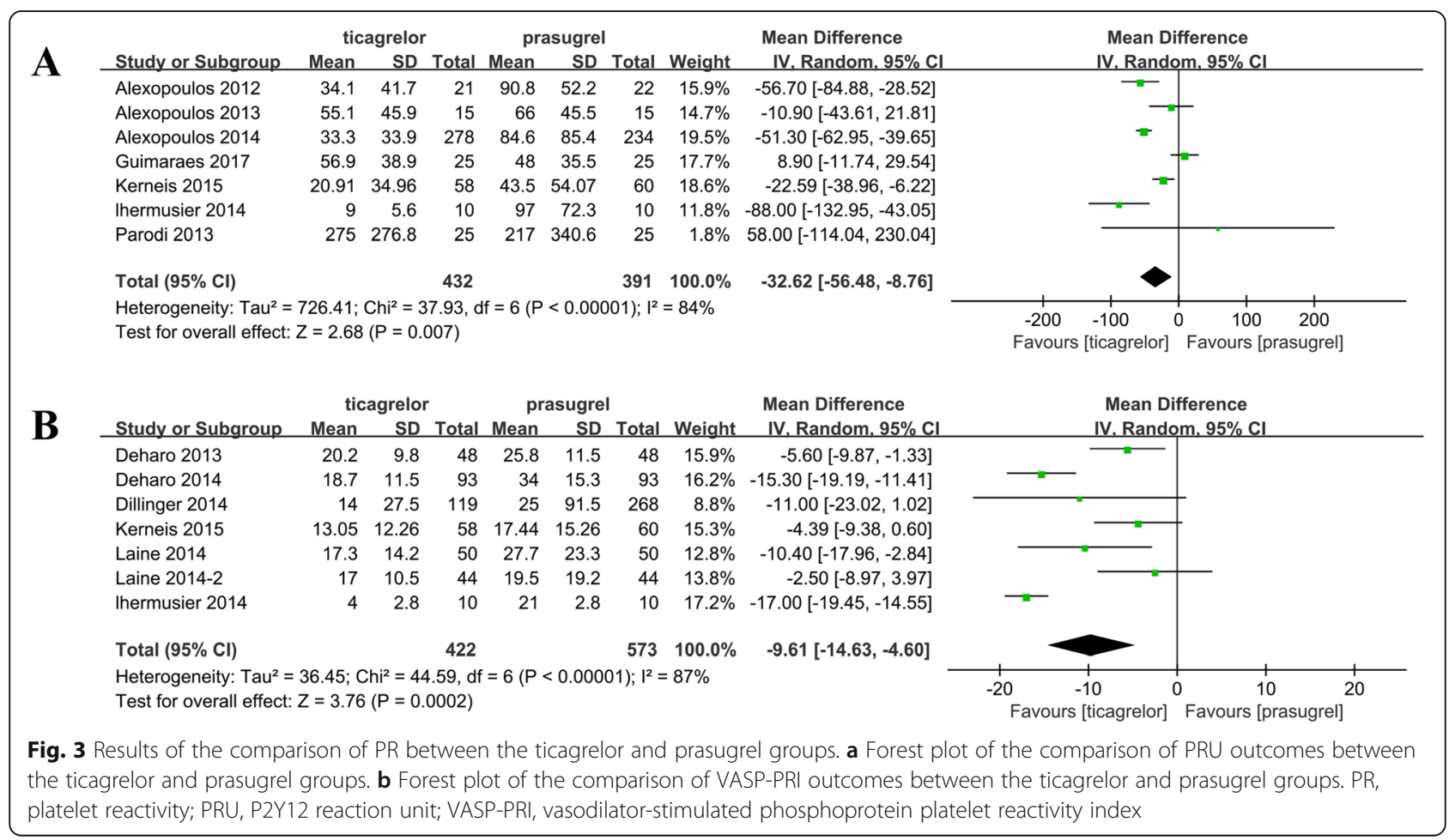


treatment initiation). The PR was high in the ACS patients with diabetes, which was treated with prasugrel $(\mathrm{WMD}=-10.40 ; 95 \% \mathrm{CI}:-17.96$ to $-2.84 ; P=0.007$; Table 3).

\section{HTPR and LTPR assessments}

Among the included studies, only two studies reported PRU-based HTPR. PRU-based HTPR was observed in $0.66 \%(2 / 305)$ of the ticagrelor-treated patients and $4.98 \%(13 / 261)$ of the prasugrel-treated patients. The pooled results revealed no significant difference between the prasugrel and ticagrelor groups $(P=0.71$; Fig. 4a), and heterogeneity was observed between studies $(P=$ $0.01, I^{2}=84 \%$ ). Moreover, five studies (all RCTs) reported HTPR based on VASP-PRI. PRI-based HTPR was observed in $2.67 \%(7 / 262)$ of the ticagrelor-treated patients and $11.45 \%(30 / 262)$ of the prasugrel-treated patients. On the basis of the pooled results, the incidence rate of HTPR in the prasugrel group was significantly higher than that in the ticagrelor group $(\mathrm{RR}=0.30 ; 95 \%$ CI: $0.12-0.75 ; P=0.01$; Fig. 4b, Table 3). Furthermore, six studies reported LTPR based on VASP-PRI, including $60.68 \%$ of the ticagrelor-treated patients and $43.51 \%$ of the prasugrel-treated patients. The results of the pooled analysis showed a lower LTPR in the prasugrel group than in the ticagrelor group ( $R R=1.40 ; 95 \% \mathrm{CI}$ : $1.08-1.81 ; P=0.01$; Fig. 4c). Subsequently, a subgroup analysis based on study types, testing time, and special population was conducted. In the HTPR (PRU) group, the analysis results were not representative because only two studies were included. In the HTPR (PRI) group, a significant difference was observed in the RCT (WMD = 0.30; 95\% CI: $0.12-0.75) ; P=0.010$ ) and $<24 \mathrm{~h}$ after the loading dose groups, which suggests the rate of HTPR was lower in the prasugrel group than in the ticagrelor group. For the rate of LTPR (PRI), a significant difference was found only at 5-30 days after treatment initiation (Table 3).

\section{Publication bias}

Egger's test was performed to evaluate the potential publication bias in the present study, and the results showed no significant bias was detected in the included studies [PR (PRU), $P=0.688$; PR (PRI), $P=0.127$; HTPR rates (PRU), $P=$ NA (the Egger test could not be performed because only two studies were included); HTPR rates (PRI), $P=0.199$; and LTPR rates (PRI), $P=0.243$.

\section{Discussion}

This meta-analysis of 14 studies compared the effects of ticagrelor and prasugrel on HTPR and LTPR according to VASP-PRI and PRU in patients with ACS. Our results showed that the ACS patients treated with prasugrel had a higher PR than those treated with ticagrelor. In addition, a lower HTPR based on VASP-PRI was found in the ACS patients treated with ticagrelor than in those treated with prasugrel. Furthermore, the results showed that a lower LTPR based on VASP-PRI was observed in the prasugrel group than in the ticagrelor group.

Ticagrelor is a cyclopentyl triazolopyrimidine that directly targets the P2Y12 platelet receptor [29]. As a novel P2Y12 blocker, ticagrelor is more potent and rapidacting than clopidogrel. The randomized PLATelet inhibition and patient Outcomes (PLATO) study showed that ticagrelor reduced the incidence rates of cardiovascular death, myocardial infarction, and stroke compared with clopidogrel [6]. In addition, ticagrelor reduces the adenosine uptake by red blood cells, decreasing its plasma concentration, and inhibits platelet aggregation [30, 31]. Prasugrel is a third-generation thienopyridine, and its active metabolite irreversibly inhibits the P2Y12 receptor by blocking its binding site, thereby inhibiting ADP-induced platelet aggregation [32]. Similarly, prasugrel also shows a clinical advantage over clopidogrel. In the Trial to Assess Improvement in Therapeutic Outcomes by Optimizing Platelet Inhibition with PrasugrelThrombolysis in Myocardial Infarction 38 (TRITONTIMI 38), prasugrel administration significantly reduced the rates of ischemic events [33]. Although both ticagrelor and prasugrel were outstanding antiplatelet agents, we revealed that prasugrel might enable a higher PR than ticagrelor. Alexopoulos et al. [7] indicated that ticagrelor produced a significantly higher platelet inhibition compared with prasugrel, which was consistent with our findings. This phenomenon was presumably explained by the fact that prasugrel irreversibly inhibited the P2Y12 receptor, whereas ticagrelor was a reversible P2Y12 blocker [34]. Moreover, the inhibition of adenosine uptake may promote the antiplatelet effects of ticagrelor. The Intracoronary Stenting and Antithrombotic Regimen: Rapid Early Action for Coronary Treatment (ISAR-REACT) 5 trial compared the impacts of ticagrelor and prasugrel on clinical events in 4000 patients [22], providing a reference based on the clinical point of view. This study suggested that ticagrelor showed a stronger antiplatelet effect than prasugrel, which was further supported our results. Unexpectedly, the study also found that the risk of myocardial infarction at 1 year after the ISAR-REACT 5 trial was significantly lower in the prasugrel group than in the ticagrelor group. Likewise, Deharo et al. [11] suggested that ticagrelor administration was associated with higher platelet inhibition and incidence of a hyper-response than prasugrel administration 1 month after ACS, which possibly exposes patients to a higher risk of bleeding complications. The assessment of end points or measurement timing might have caused this difference. 
Table 3 Outcomes of the subgroup analysis

\begin{tabular}{|c|c|c|c|c|c|}
\hline & No. of studies & Hetero & & Effect size & \\
\hline & & $\mathrm{I}^{2}(\%)$ & $\mathrm{P}_{\mathrm{H}}$ & WMD/RR $(95 \% \mathrm{Cl})$ & $P$ \\
\hline Platelet reactivity (PRU) & & & & & \\
\hline Type of studies & & & & & \\
\hline $\mathrm{RCT}$ & 5 & 86 & $<0.001$ & $-29.93(-70.21,10.36)$ & 0.15 \\
\hline Cohort & 2 & 87 & 0.005 & $-37.55(-65.66,-9.44)$ & 0.009 \\
\hline Testing time & & & & & \\
\hline$<24 \mathrm{~h}$ after the loading dose & 3 & 87 & 0.005 & $-22.48(-105.72,60.76)$ & 0.60 \\
\hline 5-30 days after initiation of treatment & 4 & 76 & 0.006 & $-36.72(-57.04,-16.40)$ & $<0.001$ \\
\hline Special Population & & & & & \\
\hline STEMI & 3 & 67 & 0.05 & $-5.77(-34.85,-23.31)$ & 0.70 \\
\hline Diabetic patients & 1 & - & - & $-10.90(-43.61,21.81)$ & 0.514 \\
\hline Platelet reactivity (PRI) & & & & & \\
\hline Type of studies & & & & & \\
\hline $\mathrm{RCT}$ & 5 & 88 & $<0.001$ & $-10.51(-16.17,-4.85)$ & $<0.001$ \\
\hline Cohort & 2 & 0 & 0.319 & $-5.36(-9.97,-0.76)$ & 0.023 \\
\hline Testing time & & & & & \\
\hline$<24 \mathrm{~h}$ after the loading dose & 3 & 89 & $<0.001$ & $-10.30(-19.70,-0.90)$ & 0.032 \\
\hline 5-30 days after initiation of treatment & 4 & 81 & $<0.001$ & $-8.91(-15.03,-2.79)$ & 0.004 \\
\hline Special Population & & & & & \\
\hline STEMI & 2 & 0 & 0.650 & $-3.69(-7.63,0.26)$ & 0.067 \\
\hline Diabetic patients & 1 & - & - & $-10.40(-17.96,-2.84)$ & 0.007 \\
\hline HTPR rates $(P R U)^{a}$ & & & & & \\
\hline Type of studies & & & & & \\
\hline $\mathrm{RCT}$ & 1 & - & - & $5.00(0.25,99.51)$ & 0.29 \\
\hline Cohort & 1 & - & - & $0.03(0.00,0.52)$ & 0.016 \\
\hline Testing time & & & & & \\
\hline 5-30 days after initiation of treatment & 2 & 84 & 0.01 & $0.39(0.001,62.72)$ & 0.71 \\
\hline HTPR rates (PRI) & & & & & \\
\hline Type of studies & & & & & \\
\hline $\mathrm{RCT}$ & 5 & 20 & 0.290 & $0.30(0.12,0.75)$ & 0.010 \\
\hline Testing time & & & & & \\
\hline$<24 \mathrm{~h}$ after the loading dose & 2 & 0 & 0.440 & $0.31(0.10,0.98)$ & 0.047 \\
\hline 5-30 days after initiation of treatment & 3 & 69 & 0.073 & $0.24(0.03,2.08)$ & 0.197 \\
\hline Special Population & & & & & \\
\hline STEMI & 1 & - & - & $0.11(0.01,2.00)$ & 0.137 \\
\hline Diabetic patients & 1 & - & - & $0.38(0.11,1.33)$ & 0.129 \\
\hline LTPR rates (PRI) & & & & & \\
\hline Type of studies & & & & & \\
\hline $\mathrm{RCT}$ & 4 & 82 & 0.0008 & $1.63(0.95,2.80)$ & 0.073 \\
\hline Cohort & 2 & 85 & 0.009 & $1.31(0.98,1.76)$ & 0.066 \\
\hline Testing time & & & & & \\
\hline$<24 \mathrm{~h}$ after the loading dose & 2 & 0 & 0.898 & $1.09(0.86,1.39)$ & 0.462 \\
\hline 5-30 days after initiation of treatment & 4 & 89 & $<0.001$ & $1.69(1.12,2.56)$ & 0.012 \\
\hline
\end{tabular}


Table 3 Outcomes of the subgroup analysis (Continued)

\begin{tabular}{|c|c|c|c|c|c|}
\hline & \multirow[t]{2}{*}{ No. of studies } & \multicolumn{2}{|c|}{ Heterogeneity test } & \multicolumn{2}{|l|}{ Effect size } \\
\hline & & $\mathrm{I}^{2}(\%)$ & $\mathrm{P}_{\mathrm{H}}$ & WMD/RR (95\% Cl) & $P$ \\
\hline \multicolumn{6}{|l|}{ Special Population } \\
\hline STEMI & 2 & 0 & 0.634 & $1.15(1.01,1.30)$ & 0.029 \\
\hline Diabetic patients & 1 & - & - & $1.11(0.79,1.56)$ & 0.545 \\
\hline
\end{tabular}

${ }^{a}$ three studies don't involved in the results of STEMI or Diabetic patients

Different individuals had various responses to antiplatelet therapy, and the residual reactivity of platelets after antiplatelet therapy was also different. Thus, testing the residual reactivity might be one of the methods to evaluate the reactivity of patients to antiplatelet therapy and the risk of thrombosis. Therefore, we compared the effects of prasugrel and ticagrelor on HTPR and LTPR. Our results showed a significant difference in HTPR according to the VASP-PRI test between the ticagrelorand prasugrel-treated groups. Notably, HTPR is influenced by different detection methods and evaluation criteria [35, 36]. However, only two studies reported PRU-based HTPR, and the results revealed no significant difference between the prasugrel and ticagrelor groups. Ferreiro et al. [37] reported that the HTPR assessed using VASP-PRI was higher than that assessed using PRU in prasugrel-treated patients. In addition, HTPR assessed using VASP-PRI is reported to be significantly lower after ticagrelor treatment than after prasugrel treatment [16]. Ticagrelor administration reduces the plasma concentration of adenosine and the activation of cyclic adenosine monophosphate-dependent protein kinases that inhibit VASP phosphorylation; however, it does not influence the PR calculated based on PRU [30, 31, 38]. The ISAR-REACT 5 trial [22] indicated that the incidence of myocardial infarction was significantly lower in prasugrel-treated patients with ACS than in ticagrelor-treated; and HTPR reduced the inhibition rate of platelet aggregation in vivo, leading to high platelet residual reactivity, which might have a higher risk of thrombotic events. In this study, we found prasugrel might enable higher PR than ticagrelor. However, the level of HTPR was lower in the patients with ACS treated with ticagrelor than in those treated with prasugrel. Our findings were inconsistent with the results of ISAR-REACT 5 trial, this inconsistency might be

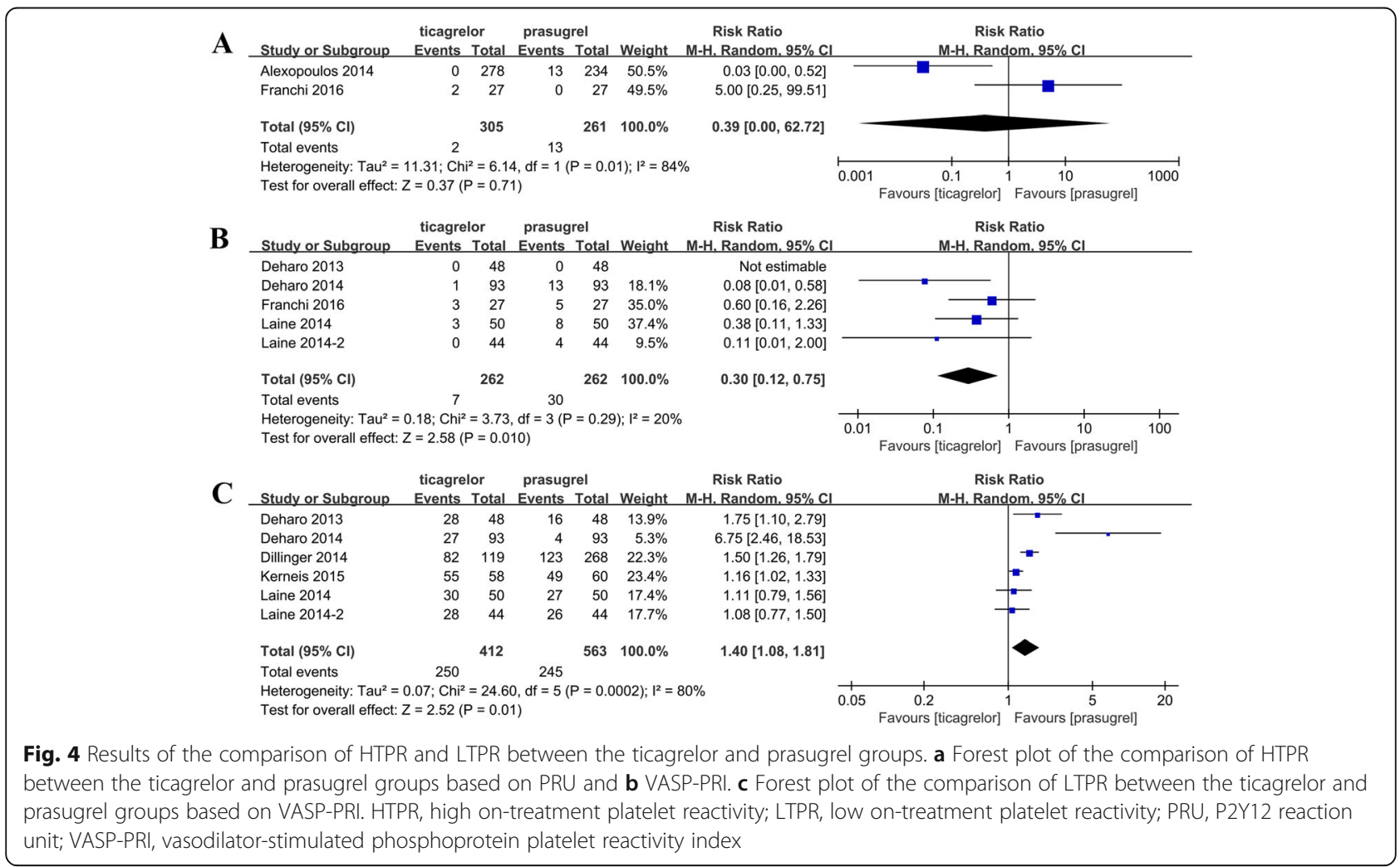


attributed to the testing methodand the sample size. Notably, HTPR is defined as a modifiable risk factor or only the marker of adverse reactions this event remaines controversial. Future studies should take this factor into account.

In addition, we found that in all the included studies, the LTPR rate was lower in the prasugrel group than in the ticagrelor group. LTPR reduced a high inhibition rate of platelet aggregation in vivo, resulting in an excessively low reactivity of platelet residues, which might lead to a higher blood risk. Observational studies have suggested an association between LTPR and bleeding, indicating that the occurrence of bleeding events in patients treated with P2Y12 receptor inhibitors is related to the excessive platelet inhibition with the consequence of LTPR [39]. Siller-Matula et al. [40] observed that $69 \%$ of the patients in the prasugrel group and $64 \%$ of the patients in ticagrelor group displayed LTPR. Another study corroborated these findings [41]. Unfortunately, our findings were inconsistent with previous studies. We proposed that the presence of confounding factors in the cohort studies might have exaggerated the difference between the two groups. Specifically, the thresholds for LTPR (VASP-PRI $<20 \%$ or $<16 \%$ ), small size (only 4 RCTs), population characteristics (ACS patients with STEMI or diabetes), timing of measurements (1 month, $6-12 \mathrm{~h}$, and other), and mean age (50, 60, or 70 years) might have confounded these findings.

This study has several limitations. First, the differences in cutoff values to define low or higher responders using VASP-PRI were not considered. Second, the study focused on PR as the primary end point and was insufficiently powered to compare clinical end points. Third, owing to the limited sample size, the subgroup analysis results were not discussed in depth. Fourth, the total number of pooled results for LTPR from the cohort studies was inconsistent with the number of pooled results from the RCTs. Therefore, additional RCTs should be examined to verify our results. Finally, ticagrelor could inhibit erythrocyte absorption of adenosine and subsequent increase in plasma adenosine concentration, which might activate A2 adenosine receptors on platelets, increase cAMP levels, and induce phosphorylation of camp-dependent protein kinases on VASP. Thus, the differences identified by VASP-PRI and PRU might be just methodological errors. These findings still need further elaboration.

\section{Conclusion}

Prasugrel might allow a higher PR than ticagrelor. Ticagrelor could lead to a decreased HTPR and increased LTPR. Several uncertainties such as the nondeterminacy of the effectiveness of ticagrelor that was estimated with the VASP-PRI or the definition of HTPR (high or modifiable risk factor) might have affected our results.

\section{Abbreviations}

ACS: Acute coronary syndrome; Cl: Confidence interval; HTPR: High ontreatment platelet reactivity; LTPR: Low on-treatment platelet reactivity; NSTEMI: Non-ST-segment elevation myocardial infarction; PCI: Percutaneous coronary intervention; PRU: P2Y12 reaction unit; RCT: Randomized controlled trial; RR: Risk ratio; STEMI: ST-segment elevation myocardial infarction; VASPPRI: Vasodilator-stimulated phosphoprotein platelet reactivity index;

WMD: Weighted mean difference

\section{Acknowledgments \\ None.}

\section{Authors' contributions}

MX-W contributed to the study conception and design. YY-Z contributed to the data acquisition. YQ-L and ZQ-S contributed to the data analysis and interpretation. LF-T contributed to the statistical analysis. XQ contributed to the data acquisition and manuscript writing. XQ-S and XL-Y contributed to the manuscript revision for important intellectual content. All authors read and approved the final manuscript.

\section{Funding}

None.

\section{Availability of data and materials}

The datasets generated and analyzed during the present study are available from the corresponding author on reasonable request.

Ethics approval and consent to participate

Not applicable.

\section{Consent for publication}

Not applicable.

\section{Competing interests}

The authors declare that they have no competing interests.

\section{Author details}

${ }^{1}$ Intensive Care Unit, Guizhou Provincial People's Hospital, No. 58 Zhongshan East Road, Nanming District, Guiyang 550002, Guizhou, China. ²Emergency Department, Guizhou Provincial People's Hospital, Guiyang 550002, Guizhou, China. ${ }^{3}$ Radiology Department, Guizhou Provincial People's Hospital, Guiyang 550002, Guizhou, China. ${ }^{4}$ Endocrine Department, The First People's Hospital of Guiyang, Guiyang 550002, Guizhou, China.

Received: 28 December 2018 Accepted: 26 June 2020

Published online: 01 October 2020

References

1. Kotecha T, Rakhit RD. Acute coronary syndromes. Clin Med. 2016;16(Suppl 6):s43-8.

2. Santos-Gallego CG, Picatoste B, Badimón JJ. Pathophysiology of acute coronary syndrome. Curr Atheroscler Rep. 2014;16(4):401.

3. Bagai A, Bhatt DL, Eikelboom JW, Mancini GJ, Cohen EA, Vijayaraghavan R, Cheema AN, Udell JA, Niznick J, Tanguay J-F. Individualizing duration of dual antiplatelet therapy after acute coronary syndrome or percutaneous coronary intervention. Circulation. 2016;133(21):2094-8.

4. Franchi F, Angiolillo DJ. Novel antiplatelet agents in acute coronary syndrome. Nat Rev Cardiol. 2015;12(1):30-47.

5. Tantry US, Bonello L, Aradi D, Price MJ, Jeong Y-H, Angiolillo DJ, Stone GW, Curzen N, Geisler T, Ten Berg J. Consensus and update on the definition of on-treatment platelet reactivity to adenosine diphosphate associated with ischemia and bleeding. J Am Coll Cardiol. 2013;62(24):2261-73.

6. Wallentin L, Becker RC, Budaj A, Cannon CP, Emanuelsson H, Held C, Horrow J, Husted S, James S, Katus H. Ticagrelor versus clopidogrel in patients with acute coronary syndromes. N Engl J Med. 2009;361(11):1045-57.

7. Alexopoulos D, Galati A, Xanthopoulou I, Mavronasiou E, Kassimis G, Theodoropoulos KC, Makris G, Damelou A, Tsigkas G, Hahalis G. Ticagrelor versus prasugrel in acute coronary syndrome patients with high onclopidogrel platelet reactivity following percutaneous coronary intervention: a pharmacodynamic study. J Am Coll Cardiol. 2012;60(3):193-9. 
8. Alexopoulos D, Stavrou K, Koniari I, Gkizas V, Perperis A, Kontoprias K, Vogiatzi C, Bampouri T, Xanthopoulou I. Ticagrelor vs prasugrel one-month maintenance therapy: impact on platelet reactivity and bleeding events. Thromb Haemost. 2014;112(3):551-7.

9. Alexopoulos D, Xanthopoulou I, Mavronasiou E, Stavrou K, Siapika A, Tsoni E, Davlouros P. Randomized assessment of ticagrelor versus prasugrel antiplatelet effects in patients with diabetes. Diabetes Care. 2013;36(8):2211-6.

10. Angiolillo DJ, Curzen N, Gurbel P, Vaitkus P, Lipkin F, Li W, Jakubowski JA, Zettler M, Effron MB, Trenk D. Pharmacodynamic evaluation of switching from ticagrelor to prasugrel in patients with stable coronary artery disease: results of the SWAP-2 study (switching anti Platelet-2). J Am Coll Cardiol. 2014;63(15):1500-9.

11. Deharo P, Bassez C, Bonnet G, Pankert M, Quilici J, Lambert M, Verdier V, Morange $\mathrm{P}$, Alessi $\mathrm{M}-\mathrm{C}$, Bonnet J-L. Prasugrel versus ticagrelor in acute coronary syndrome: a randomized comparison. Int J Cardiol. 2013;170(2): e21-2.

12. Deharo P, Pankert M, Bonnet G, Quilici J, Bassez C, Morange P, Alessi M-C, Bonnet J-L, Cuisset T. Body mass index has no impact on platelet inhibition induced by ticagrelor after acute coronary syndrome, conversely to prasugrel. Int J Cardiol. 2014;176(3):1200-2.

13. Dillinger J, Manzo SS, Amsallem M, Sideris G, Voicu S, Henry P, Drouet L. Ticagrelor effectiveness overestimated by VASP index: platelet inhibition by ticagrelor versus prasugrel in acute coronary syndrome patients according to platelet function tests. Int J Cardiol. 2014;176(2):557.

14. Franchi F, Faz GT, Rollini F, Park Y, Cho JR, Thano E, Hu J, Kureti M, Aggarwal $N$, Durairaj A. Pharmacodynamic effects of switching from Prasugrel to Ticagrelor: results of the prospective, randomized SWAP-3 study. J Am Coll Cardiol Intv. 2016;9(11):1089-98.

15. Kerneis M, Silvain J, Abtan J, Hauguel M, Barthélémy O, Payot L, Brugier D, Galier S, Collet J-P, Montalescot G. Platelet effect of prasugrel and ticagrelor in patients with ST-segment elevation myocardial infarction. Arch Cardiovasc Dis. 2015;108(10):502-10.

16. Laine M, Frere $C$, Toesca $R$, Berbis J, Barnay $P$, Pansieri M, Michelet $P$, Bessereau J, Camilleri E, Ronsin O. Ticagrelor versus prasugrel in diabetic patients with an acute coronary syndrome. A pharmacodynamic randomised study. Thromb Haemost. 2014;111(2):273-8.

17. Laine M, Gaubert M, Frère C, Peyrol M, Thuny F, Yvorra S, Chelini V, Bultez B, Luigi S, Mokrani Z. COM parison of $\mathrm{P}$ latelet reactivity following pr a sugrel and ticagrelor loading dose in S T-S egment elevation myocardial infarctl ON patients: the COMPASSION study. Platelets. 2015;26(6):570-2.

18. Lhermusier T, Voisin S, Murat G, Mejean S, Garcia C, Bataille V, Lipinski M, Carrie D, Sie P. Switching patients from clopidogrel to novel P2Y12 receptor inhibitors in acute coronary syndrome: comparative effects of prasugrel and ticagrelor on platelet reactivity. Int J Cardiol. 2014;174(3):874-6.

19. Parodi G, Valenti R, Bellandi B, Migliorini A, Marcucci R, Comito V, Carrabba N, Santini A, Gensini GF, Abbate R. Comparison of prasugrel and ticagrelor loading doses in ST-segment elevation myocardial infarction patients: RAPID (Rapid activity of platelet inhibitor drugs) primary PCI study. J Am Coll Cardiol. 2013;61(15):1601-6.

20. Sardella G, Calcagno S, Mancone M, Lucisano L, Pennacchi M, Stio RE, Placentino F, Di Roma A, Cavallo E, Palmirotta R. Comparison of therapy with Ticagrelor, Prasugrel or high Clopidogrel dose in $\mathrm{PCl}$ patients with high on treatment platelet reactivity and genotype variation. TRIPLETE RESET trial. Int J Cardiol. 2015;194:60-2.

21. Yudi MB, Clark DJ, Farouque O, Eccleston D, Andrianopoulos N, Duffy SJ, Brennan A, Lefkovits J, Ramchand J, Yip T. Clopidogrel, prasugrel or ticagrelor in patients with acute coronary syndromes undergoing percutaneous coronary intervention. Intern Med J. 2016;46(5):559-65.

22. Schüpke S, Neumann F-J, Menichelli M, Mayer K, Bernlochner I, Wöhrle J, Richardt G, Liebetrau C, Witzenbichler B, Antoniucci D. Ticagrelor or prasugrel in patients with acute coronary syndromes. N Engl J Med. 2019; 381(16):1524-34

23. Lemesle G, Schurtz G, Bauters C, Hamon M. High on-treatment platelet reactivity with ticagrelor versus prasugrel: a systematic review and metaanalysis. J Thromb Haemost. 2015;13(6):931-42.

24. Zhang $H$, Zhang $P$, Dong $P$, Yang $X$, Wang $Y$, Zhang $H$, Yan J, Zhang $Y$ Zhang T, Li Y. Effect of ticagrelor versus prasugrel on platelet reactivity: a meta-analysis. Coron Artery Dis. 2017;28(7):597-604.

25. Moher D, Liberati A, Tetzlaff J, Altman DG. Preferred reporting items for systematic reviews and meta-analyses: the PRISMA statement. Ann Intern Med. 2009;151(4):264-9.
26. Well G, Shea B, O'COnnell D. The Newcastle-Ottawa Scale (NOS) for assessing the quality of nonrandomised studies in meta-analyses. Ottawa Heath Research Institute. In: 3rd Symposium on Systematic Reviews: Beyond the Basics Oxford, Engiand, vol. 2000; 2000.

27. Higgins JP, Thompson SG, Deeks JJ, Altman DG. Measuring inconsistency in meta-analyses. Br Med J. 2003;327(7414):557-60.

28. Guimarães LF, Généreux P, Silveira D, Pesaro AE, Falcão F, Barbosa BR, de Souza CF, Fonseca FA, Alves CM, Carvalho AC, Stone GW, Caixeta A. P2Y12 receptor inhibition with prasugrel and ticagrelor in STEMI patients after fibrinolytic therapy: analysis from the SAMPA randomized trial. Int J Cardiol. 2017;230:204-8.

29. Husted S, Emanuelsson H, Heptinstall S, Sandset PM, Wickens M, Peters G. Pharmacodynamics, pharmacokinetics, and safety of the oral reversible P2Y12 antagonist AZD6140 with aspirin in patients with atherosclerosis: a double-blind comparison to clopidogrel with aspirin. Eur Heart J. 2006;27(9): 1038-47.

30. Bonello L, Laine M, Kipson N, Mancini J, Helal O, Fromonot J, Gariboldi V, Condo J, Thuny F, Frere C. Ticagrelor increases adenosine plasma concentration in patients with an acute coronary syndrome. J Am Coll Cardiol. 2014;63(9):872-7.

31. Linden MD, Barnard MR, Frelinger AL, Michelson AD, Przyklenk K. Effect of adenosine a 2 receptor stimulation on platelet activation-aggregation: differences between canine and human models. Thromb Res. 2008;121(5): 689-98.

32. Jernberg T, Payne CD, Winters KJ, Darstein C, Brandt JT, Jakubowski JA, Naganuma H, Siegbahn A, Wallentin L. Prasugrel achieves greater inhibition of platelet aggregation and a lower rate of non-responders compared with clopidogrel in aspirin-treated patients with stable coronary artery disease. Eur Heart J. 2006;27(10):1166-73.

33. Ederhy S. TRITON-TIMI 38 investigators. Prasugrel versus clopidogrel in patients with acute coronary syndromes. MT Cardiol. 2007;3(6):441-3.

34. Stone GW. Ticagrelor in ACS: redefining a new standard of care? Lancet. 2010:375(9711):263-5.

35. Lemesle G, Landel J-B, Bauters A, Delhaye C, Bonello L, Sudre A, Susen S, Bauters C, Lablanche J-M. Poor agreement between light transmission aggregometry, Verify now P2Y12 and vasodilatator-stimulated phosphoprotein for clopidogrel low-response assessment: a potential explanation of negative results of recent randomized trials. Platelets. 2014; 25(7):499-505.

36. Bonello L, Tantry US, Marcucci R, Blindt R, Angiolillo DJ, Becker R, Bhatt DL, Cattaneo M, Collet JP, Cuisset T. Consensus and future directions on the definition of high on-treatment platelet reactivity to adenosine diphosphate. J Am Coll Cardiol. 2010;56(12):919-33.

37. Ferreiro JL, Ueno M, Tello-Montoliu A, Tomasello SD, Seecheran N, Desai B, Rollini F, Guzman LA, Bass TA, Angiolillo DJ. Impact of prasugrel reload dosing regimens on high on-treatment platelet reactivity rates in patients on maintenance prasugrel therapy. J Am Coll Cardiol Intv. 2013;6(2):182-4.

38. Gessi S, Varani K, Merighi S, Ongini E, Borea P. A2A adenosine receptors in human peripheral blood cells. Br J Pharmacol. 2000;129(1):2-11.

39. Sibbing D, Schulz S, Braun S, Morath T, Stegherr J, Mehilli J, Schömig A, Von Beckerath N, Kastrati A. Antiplatelet effects of clopidogrel and bleeding in patients undergoing coronary stent placement. J Thromb Haemost. 2010; 8(2):250-6.

40. Siller-Matula JM, Akca B, Neunteufl T, Maurer G, Lang IM, Kreiner G, Berger R, Delle-Karth $\mathrm{G}$. Inter-patient variability of platelet reactivity in patients treated with prasugrel and ticagrelor. Platelets. 2016;27(4):373-7.

41. Bernlochner I, Mayer K, Orban M, Morath T, Jaitner J, Rössner L, Gross L, Laugwitz K-L, Kastrati A, Sibbing D. Ticagrelor versus prasugrel in patients with high on-clopidogrel treatment platelet reactivity after PCl: the ISARADAPT-PF study. Platelets. 2016;27(8):796-804.

\section{Publisher's Note}

Springer Nature remains neutral with regard to jurisdictional claims in published maps and institutional affiliations. 